\title{
Effects of metformin and sitagliptin on glycolipid metabolism in type 2 diabetic rats on different diets
}

Juhong Yang, Tu Ba, Liming Chen, Chunyan Shan, Miaoyan Zheng, Ying Wang, Huizhu Ren, Jingli Chen, Jie Xu, Fei Han, Yi Zhang, Xiaoyun Yang, Baocheng Chang

Key Laboratory of Hormone and Development (Ministry of Health), Tianjin Key Laboratory of Metabolic Disease, Tianjin Hospital and Tianjin Institute of Endocrinology, Tianjin Medical University, Tianjin, China

Submitted: 28 March 2014

Accepted: 7 July 2014

Arch Med Sci 2016; 12, 2: 233-242

DOI: 10.5114 /aoms.2016.59249

Copyright (c) 2016 Termedia \& Banach

\section{Abstract}

Introduction: The aim of the study was to investigate the effects of metformin and sitagliptin on glycolipid metabolism in type 2 diabetes after different diets.

Material and methods: Seventy Male Sprague Dawley rats were fed with a high fat diet followed by streptozotocin treatment to induce type $2 \mathrm{di}$ abetes. Then all rats were randomly divided into a control group, a metformin group $(200 \mathrm{mg} / \mathrm{kg})$, and a sitagliptin group $(10 \mathrm{mg} / \mathrm{kg})$. Each group was further divided into 4 groups receiving one load of high carbohydrate diet ( $45 \%$ glucose, $4.5 \mathrm{ml} / \mathrm{kg}$ ), high fat diet ( $20 \%$ lipid emulsion, $4.5 \mathrm{ml} / \mathrm{kg}$ ), high protein diet $(20 \%$ whey protein, $10 \mathrm{ml} / \mathrm{kg}$ ) or mixed meal, respectively. The caloric densities were all $33 \mathrm{~kJ} / \mathrm{kg}$. Postprandial blood glucose (P2BG), triglyceride (TG), glucagon-like peptide-1 (GLP-1), glucagon and insulin levels were measured.

Results: In the high carbohydrate group, sitagliptin was more efficient in lowering P2BG compared with metformin $(p<0.05)$. In the high-fat group, metformin was more powerful in lowering TG $(p<0.05)$ and P2BG $(p<0.05)$ levels because of its improvement of insulin sensitivity. In the high protein diet group, metformin did not reduce the P2BG level $(p>0.05)$, although it did reduce the TG level $(p<0.05)$. In the mixed diet group, metformin was more efficient in lowering P2BG $(p<0.05)$ but had a similar effect on TG $(p>0.05)$ compared with sitagliptin.

Conclusions: In the type 2 diabetic model, metformin and sitagliptin have different effects on glycolipid metabolism after different diets. If it is proved in type 2 diabetic patients, then different medicines may be recommended according to different diets in order to improve glycolipid metabolism.

Key words: type 2 diabetes, diet intervention, glycolipid metabolism, metformin, sitagliptin.

\section{Introduction}

With the rapid development of the economy, diabetes mellitus has become an important public health problem worldwide. According to figures released by the International Diabetes Federation (IDF), in 2011 there were 366 million people with diabetes, and this is expected to rise to 552 million by 2030 . Most people with diabetes live in low- and middle-income countries, and these countries will also see the greatest increase over the next 19 years [1, 2]. Approximately 5.1 million people aged between

\author{
Corresponding author: \\ Baocheng Chang PhD \\ Tianjin Institute \\ of Endocrinology \\ Tianjin Medical University \\ No. 66, Tong'an Road \\ Heping District \\ 300070 Tianjin, China \\ Phone: +862223333211 \\ E-mail: \\ changbc1970@126.com
}


20 and 79 years died from diabetes in 2013 , accounting for $8.4 \%$ of global all-cause mortality among people in this age group. Global health spending to treat diabetes and manage complications totaled at least USD 548 billion in 2013. By 2035, this number is projected to exceed USD 627 billion $[3,4]$.

Although type 2 diabetes is a multi-genetic disease, and many genes have a close relationship with type 2 diabetes such as TCF7L2, peroxisome proliferator-activated receptor $\gamma$ (PPAR $\gamma$ ), S-transferase P1, etc, environmental factors such as high fat diet play more important roles in the development of this disease [5-7]. Medical nutrition therapy (MNT) for the management of diabetes plays an important role in preventing complications associated with diabetes, especially the management of metabolic control and optimal weight $[8,9]$. The American Diabetes Association recommends that diabetes treatment should include a balanced diet low in fat and carbohydrate and a reduction in calories, in order to reduce cardiovascular risk factors and increase insulin sensitivity $[10,11]$. But diet intervention is extremely difficult, and there is a lack of compliance for most of our patients. Different patient has different eating habit, and in some situations patients tend to eat unbalanced food with an irrational proportion of diet ingredients, such as a high fat diet, a high protein diet or a high carbohydrate diet. These unbalanced food will cause insulin resistance and weight gain, and finally increase the risk of cardiovascular diseases $[12,13]$. Therefore, how to minimize the harmful effects of these irrational diets is of great importance. Until now, different hypoglycemic agents are increasingly developed to treat type 2 diabetes including sulfonylureas, thiazolidinediones, non-sulfonylurea insulin secretagogues, metformin, glycosidase inhibitors, and newly developed medicines (such as glucagon-like peptide-1 (GLP-1) analogues, dipeptidyl peptidase(DPP)-IV inhibitors, sodium-glucose co-transporter 2 inhibitor) [14-17]. The classes vary by mechanism of action, and may offer specific advantages that make it possible to use these medicines to improve glycolipid metabolism according to different diets. In the present study, we first analyzed the characteristics of glycolipid metabolism of different diets (high protein diet, high fat diet, high protein diet or mixed diet), then we compared the intervention effects of metformin (as a traditional medicine) and sitagliptin (as a new medicine) on glycolipid metabolism in type 2 diabetic (T2DM) rats on different diets.

\section{Material and methods}

\section{Animals and protocol}

Type 2 diabetes mellitus was induced in male Sprague Dawley rats (7-8 weeks of age, 200-250 g) using streptozotocin (STZ) according to $\mathrm{Bi}$ et al. [17]. Briefly, the rats were fed for 8 weeks with a high-fat diet (63\% of calories as fat), which was prepared by supplementing normal chow with $10 \%(\mathrm{w} / \mathrm{w})$ lard, $10 \%(\mathrm{w} / \mathrm{w})$ sucrose, $1 \%(\mathrm{w} / \mathrm{w})$ cholesterol, and $0.3 \% \mathrm{w} / \mathrm{w}$ bile acid (sodium salt). This was followed by a single low-dosage i.p. injection of STZ (30 mg/kg; Sigma) after 12-h fast. Plasma glucose in diabetic rats increased to at least $16.7 \mathrm{mmol} / \mathrm{l}(300 \mathrm{mg} / \mathrm{dl})$ within 3 days after STZ injection and remained at least at this level throughout the experiment. A group of nondiabetic rats fed solely on chow diet was used as the control.

\section{Grouping and intervention}

Rats were randomly divided into a control group ( $n=20)$, a metformin intervention group $(n=20)$ and a sitagliptin intervention group $(n=20)$. Then each group was further divided into a high carbohydrate group ( $n=5)$, a high fat group $(n=5)$, a high protein group $(n=5)$ and a mixed meal group $(n=5)$. Briefly, after fasting for $14 \mathrm{~h}$, rats in metformin groups were gavaged by $200 \mathrm{mg} / \mathrm{kg}$ metformin combined with $45 \%$ glucose (4.5 ml/kg), 20\% lipid emulsion $(4.5 \mathrm{ml} / \mathrm{kg})$, $20 \%$ whey protein $(10 \mathrm{ml} / \mathrm{kg})$ or mixed meal ( $1: 1: 1$ of above diet ingredients). The ingredients of lipid emulsion were: $20 \%$ soybean oil, $1.2 \%$ egg yolk phospholipids and $2.25 \%$ glycerin. The ingredients of whey protein were: $\alpha$-lactalbumin $11.3-$ $16.5 \%$, $\beta$-lactoglobulin 38-49\%, immunoglobulin 5.0-8.0\%, glycomacropeptides $15.0-20.0 \%$, lactoferrin $1.3-1.8 \%$, bovine serum albumin $3.0-5.0 \%$. Rats in sitagliptin groups were gavaged by $10 \mathrm{mg} /$ $\mathrm{kg}$ sitagliptin combined with $45 \%$ glucose $(4.5 \mathrm{ml} /$ $\mathrm{kg}), 20 \%$ lipid emulsion ( $4.5 \mathrm{ml} / \mathrm{kg}), 20 \%$ whey protein $(10 \mathrm{ml} / \mathrm{kg})$ or mixed meal (1: 1: 1 of above diet ingredients). All diets used were isocaloric with a caloric density of $33 \mathrm{~kJ} / \mathrm{kg}$.

Fasting and postprandial blood glucose (P2BG) were measured from the tail vein using a glucose meter (Johnson \& Johnson Medical Ltd, New Brunswick, NJ, USA); and venous blood was obtained from the inner canthus to determine levels of triglyceride (TG), GLP-1, glucagon and insulin by enzyme-linked immunosorbent assay (ELISA, Assay Biotechnology, USA). Homeostasis model assessment insulin resistance (HOMA-IR) was calculated as an indicator of insulin resistance according to the formula: HOMA-IR = fasting glucose $[\mathrm{mM}] \times$ fasting insulin $[\mathrm{mU} / \mathrm{ml}] / 22.5$.

This study was approved by the Tianjin Medical University Animal Committee, and the rats were maintained in accordance with the Tianjin Medical University guidelines for the care and use of laboratory animals.

\section{Statistical analysis}

Results are presented as the mean and S.E.M. The statistical significance of differences was an- 
alyzed by one-way ANOVA followed by post hoc Bonferroni's multiple comparison tests or Kruskal-Wallis for nonparametric data followed by Dunn's multiple comparison tests. The analyses were performed using SPSS for Windows version 17.0 , and $p<0.05$ was considered to be statistically significant.

\section{Results}

The plasma glucose in diabetic rats increased greatly compared with normal rats $(24.0 \pm 3.1$ vs. $5.1 \pm 0.3 \mathrm{mmol} / \mathrm{l}, p<0.01) 3$ days after STZ injection, and was stabilized at a steady high level after 7 days. Compared with normal rats, diabetic rats had a significantly higher level of blood glucose (27.7 \pm 1.9 vs. $5.4 \pm 0.4 \mathrm{mmol} / \mathrm{l}, p<0.01$ ), plasma insulin (42.34 \pm 6.31 vs. $18.11 \pm 4.36 \mathrm{mU} / \mathrm{ml}, p<0.01)$, and HOMA-IR (54.59 \pm 13.93 vs. $4.15 \pm 1.01, p<0.01)$.

\section{Characteristics of postprandial glycolipid} metabolism after one load of different diets

Compared with fasting glucose, P2BG increased greatly after a high carbohydrate diet $(22.50 \pm 1.04$ vs. $29.82 \pm 2.52 \mathrm{mmol} / \mathrm{l}, p<0.01)$ and a high fat $\operatorname{diet}(23.12 \pm 2.17$ vs. $29.90 \pm 3.96 \mathrm{mmol} / \mathrm{l}, p<0.01)$, but not in the high protein diet $(21.98 \pm 1.00 \mathrm{vs}$. $22.46 \pm 1.48 \mathrm{mmol} / \mathrm{l}, p>0.05)$ and mixed diet groups $(22.72 \pm 2.04$ vs. $23.29 \pm 2.39 \mathrm{mmol} / \mathrm{l}$, $p>0.05)$. $\mathrm{P} 2 \mathrm{BG}$ in the high carbohydrate diet and high fat diet groups was higher than that in the high protein group and mixed group, but there was no difference between the high carbohydrate group and high fat group. In the high protein group, P2BG was lower compared with other groups (Figure 1).

The postprandial TG level in the high lipid group was higher than that in other groups, and there was no difference between the high protein group, the high carbohydrate group and the mixed group. The GLP-1 did not differ between different groups. Glucagon in the high protein group was significantly higher than that in other groups, followed by the high carbohydrate group, the mixed diet group, and the high lipid group. Insulin level in the high lipid group was higher than that in other groups, followed by the high protein group and the high carbohydrate group, and no difference was found between the high carbohydrate diet and the mixed diet (Figure 2).

\section{Effects of metformin and sitagliptin on glycolipid metabolism after different diets}

\section{Effects of metformin and sitagliptin on glycolipid metabolism after high carbohydrate diet}

Rats in the high-carbohydrate group had a higher level of glucagon but lower level of insulin among the four diet groups. P2BG but not TG was decreased in the metformin intervention group. Glucagon level was decreased whereas GLP-1 and insulin level were not changed by metformin treatment. Sitagliptin was efficient in lowering the P2BG by inhibiting the glucagon level and increasing insulin and GLP-1 secretion. Triglyceride did not change after sitagliptin intervention in the high carbohydrate group. Sitagliptin treatment led to a greater decrease of P2BG and glucagon, and a greater increase of insulin and GLP-1 secretion compared with metformin. No difference was found for TG between the metformin and sitagliptin group (Figure 3).

\section{Effects of metformin and sitagliptin on glycolipid metabolism after high fat diet}

In the high-fat group, postprandial glucose and TG level were the highest in the four groups, whereas its insulin level was also the highest in the four groups. Metformin was efficient in lowering P2BG and TG level. Insulin level was also decreased after metformin treatment. The GLP-1 and glucagon did not change after metformin intervention. Sitagliptin was efficient in lowering P2BG and TG levels. Sitagliptin treatment also led to an increase of GLP-1 but a decrease of glucagon. Insulin was not changed after sitagliptin treatment. Sitagliptin treatment led to a greater decrease of P2BG, but metformin treatment caused a greater reduction of TG level. In the meantime, insulin was lower in the metformin group than in the sitagliptin group (Figure 4).

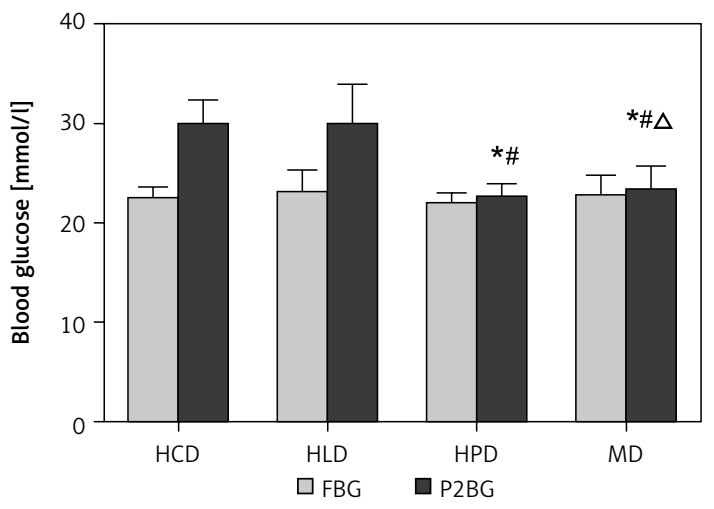

Figure 1. Postprandial blood glucose in type 2 diabetic rats receiving one load of different diets. After fasting for $14 \mathrm{~h}$, type 2 diabetic rats were gavaged respectively by $45 \%$ glucose $(4.5 \mathrm{ml} / \mathrm{kg}$, HCD group), $20 \%$ lipid emulsion (4.5 ml/kg, HLD group), $20 \%$ lactalbumin (10 ml/kg, HPD group) or mixed meal (1:1:1 of above diet ingredients, MD group). The energy was $33 \mathrm{~kJ}$ for all the diets

$H C D$ - high carbohydrate diet, HLD - high lipid diet, HPD - high protein diet, $M D$ - mixed diet, FBG - fasting blood glucose, P2BG - postprandial blood glucose, *compared with HCD group $p<0.05$; "compared with HLD group $p<0.05,{ }^{4}$ compared with HPD group $p<0.05$. 
A

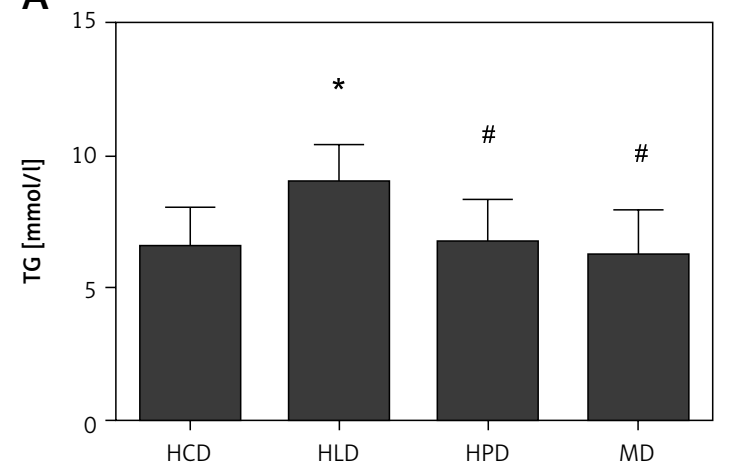

C

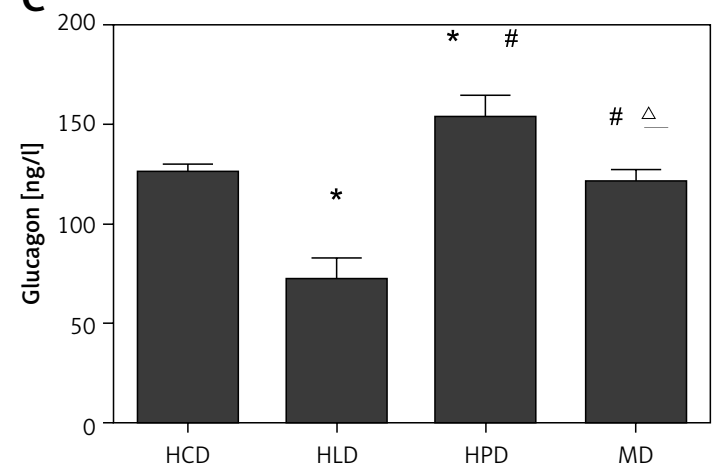

B

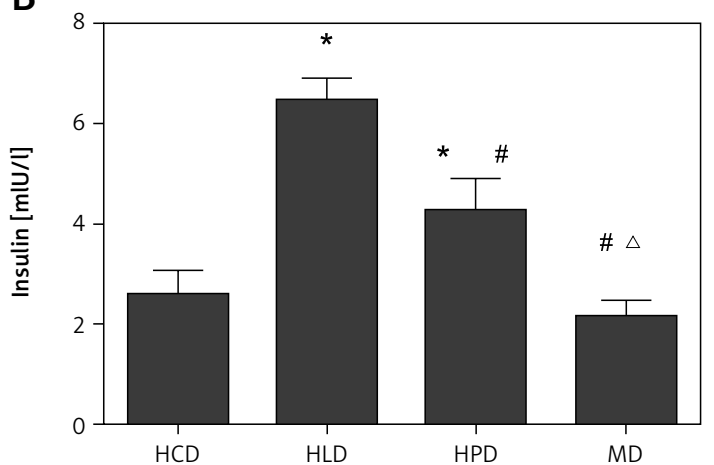

D

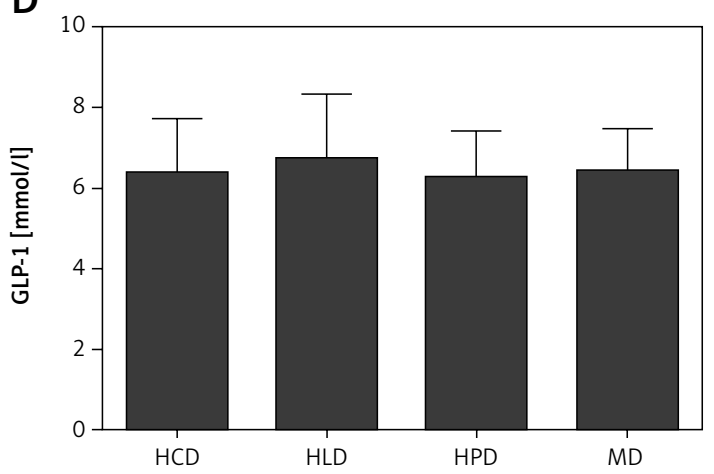

Figure 2. Postprandial blood TG (A), insulin (B), glucagon (C), and GLP-1 (D) level in type 2 diabetic rats receiving one load of different diets. After fasting for $14 \mathrm{~h}$, type 2 diabetic rats were gavaged respectively by $45 \%$ glucose (4.5 ml/kg, HCD group), 20\% lipid emulsion ( $4.5 \mathrm{ml} / \mathrm{kg}$, HLD group), 20\% lactalbumin (10 ml/kg, HPD group) or mixed diet (1:1:1 of above diet ingredients, MD group). The energy was $33 \mathrm{~kJ}$ for all the diets

$H C D$ - high carbohydrate diet, HLD - high lipid diet, HPD - high protein diet, MD - mixed diet, * compared with HCD group $p<0.05,{ }^{*}$ compared with HLD group $p<0.05 ;{ }^{4}$ compared with HPD group $p<0.05$.

\section{Effects of metformin and sitagliptin on}

glycolipid metabolism after high protein diet

In the high-protein group, the postprandial glucose and lipid levels were the lowest among the four groups. The glucagon level in this group was higher than in other groups. Metformin had no effect on P2BG after a high protein diet compared with the control group, although it reduced the level of TG. Metformin did not change the level of GLP-1 and glucagon. Insulin decreased in the metformin intervention group after a high protein diet. Sitagliptin significantly reduced the level of P2BG, but had no effect on TG level. Moreover, sitagliptin increased the level of GLP-1 and insulin, but reduced the level of glucagon. After a high protein diet, sitagliptin was more efficient in lowering P2BG, whereas metformin was more efficient in lowering TG. The insulin level in the metformin group was lower than that in the sitagliptin group (Figure 5).

\section{Effects of metformin and sitagliptin on glycolipid metabolism after mixed diet}

After a mixed diet, the P2BG was lower than the high carbohydrate group and the high lipid group, and higher than the high protein group; the
TG level was lower than other groups. Metformin treatment reduced P2BG and TG significantly. Metformin also reduced the level of glucagon and insulin, although it did not change the level of GLP-1. Sitagliptin treatment reduced P2BG and TG significantly. Sitagliptin also reduced the glucagon level, but increased the insulin level after a mixed diet. After a mixed diet, metformin was more powerful in lowering P2BG but had a similar effect on TG compared with sitagliptin. Glucagon decreased more in the sitagliptin group, whereas insulin decreased more in the metformin group. GLP-1 did not differ between these two groups (Figure 6).

\section{Discussion}

Challenged by the high prevalence of type 2 diabetes, different hypoglycemic agents are increasingly developed to treat type 2 diabetic patients, including sulfonylureas, thiazolidinediones, non-sulfonylurea insulin secretagogues, metformin, glycosidase inhibitors, and newly developed medicine such as GLP-1 analogues, DPP-IV inhibitors, and sodium-glucose co-transporter 2 inhibitor [18-21]. Until now, large studies have focused on the effects of different medicines on 
A

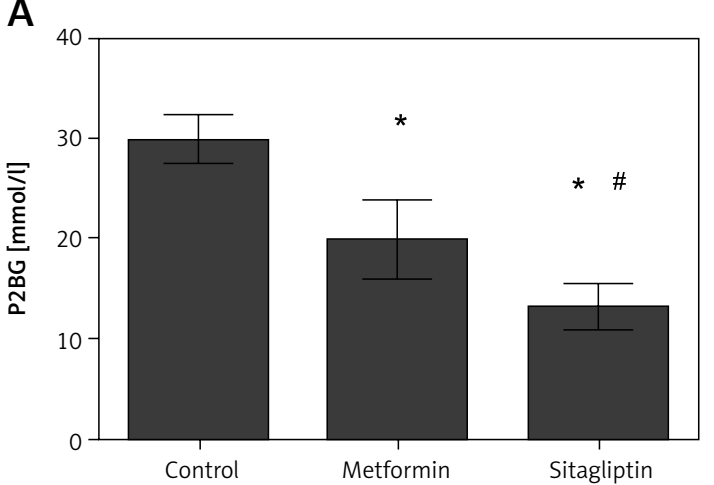

C

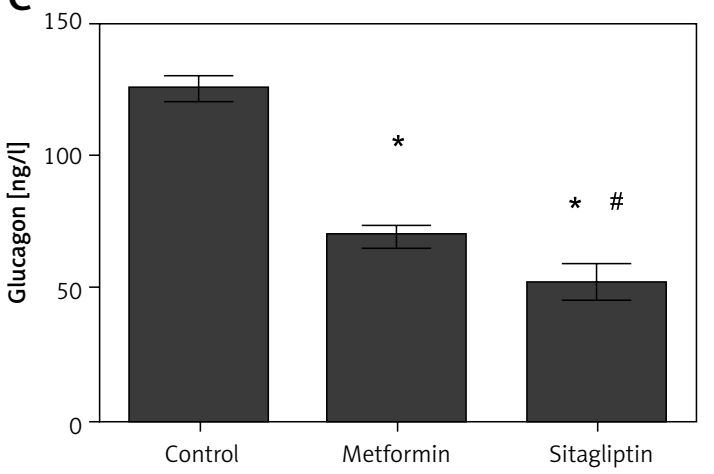

E

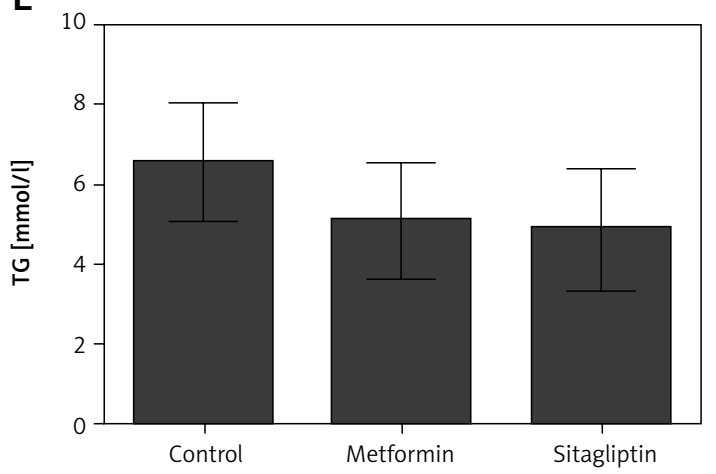

glycolipid metabolism and their underlying mechanism, but less have compared their effects in patients with different diet structures or taking different food. In a recently published systematic meta-analysis, the investigators found that the hypoglycemic effect of acarbose is superior in patients consuming an Eastern diet than in those consuming a Western diet and is similar to that of sulfonylureas, metformin, and glinide drugs [22]. However, because the author found no study that has directly compared patients consuming different types of diet, they used fixed-and random-effect models to indirectly compare the hypoglycemic effect of acarbose monotherapy with that of placebo and/or comparator drugs in patients. Therefore, our study is important because we for the first time directly compared the effect of metformin and sitagliptin on glycolipid metabolism in type 2 diabetic rats on different diets.

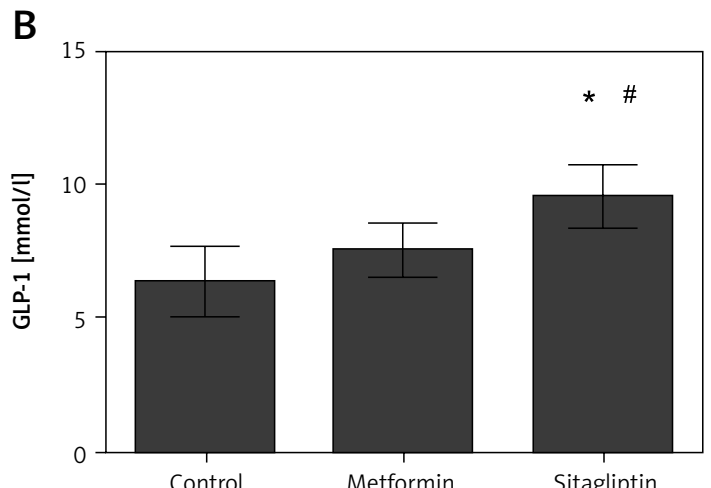

D

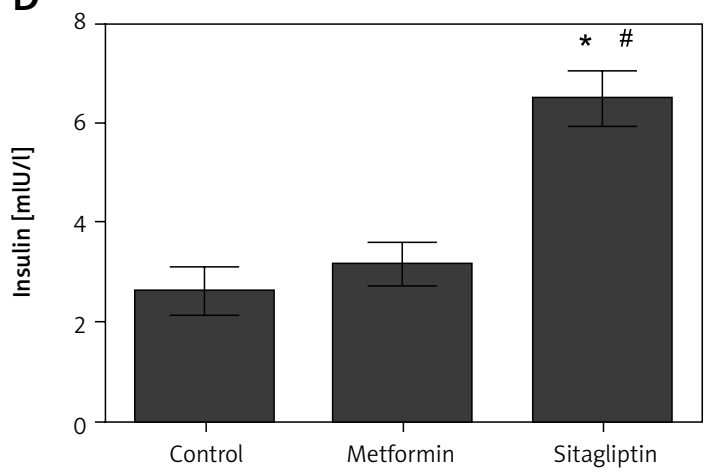

Figure 3. Effects of metformin and sitagliptin on glycolipid metabolism after one load of high carbohydrate diet. Rats in metformin groups were gavaged by $200 \mathrm{mg} / \mathrm{kg}$ metformin or $10 \mathrm{mg} / \mathrm{kg}$ sitagliptin before being gavaged by $45 \%$ glucose (4.5 ml/kg, HCD group), $20 \%$ lipid emulsion (4.5 ml/kg, HLD group), 20\% lactalbumin (10 ml/kg, HPD group) or mixed diet (1:1:1 of above diet ingredients, MD group). The energy was $33 \mathrm{~kJ}$ for all the diets

$H C D$ - high carbohydrate diet, HLD - high lipid diet, $H P D$ - high protein diet, MD - mixed diet; *compared with control group $p<0.05$; "compared with metformin group $p<0.05$.

In our study, we found that P2BG level decreased more in the sitagliptin group than in the metformin group after a high carbohydrate diet. The underlying mechanism is not clear. As shown in our study, rats in the high-carbohydrate group had a higher level of glucagon but a lower level of insulin among the four diet groups, and sitagliptin had more profound effects on glucagon inhibition and GLP-1/insulin secretion compared with metformin, which might explain its superiority in controlling P2BG after a high carbohydrate diet. Sitagliptin is an oral anti-diabetic drug of the DPP-IV inhibitor class. It works to competitively inhibit the enzyme DPP-IV, which breaks down the incretins GLP-1 and gastric inhibitory polypeptide, gastrointestinal hormones released in response to a meal. They are able to increase the secretion of insulin and suppress the release of glucagon by the $\alpha$ cells of the pancreas [23]. 
A

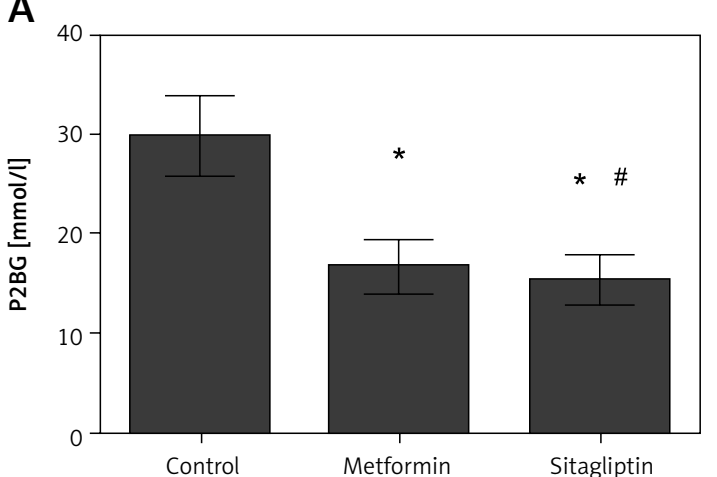

C

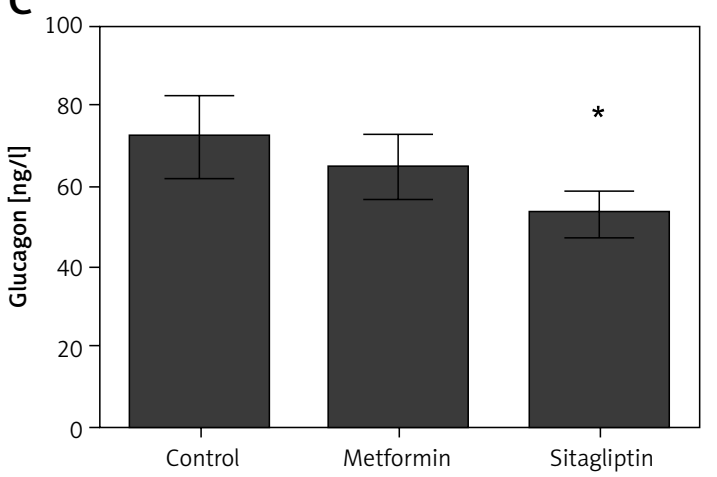

E

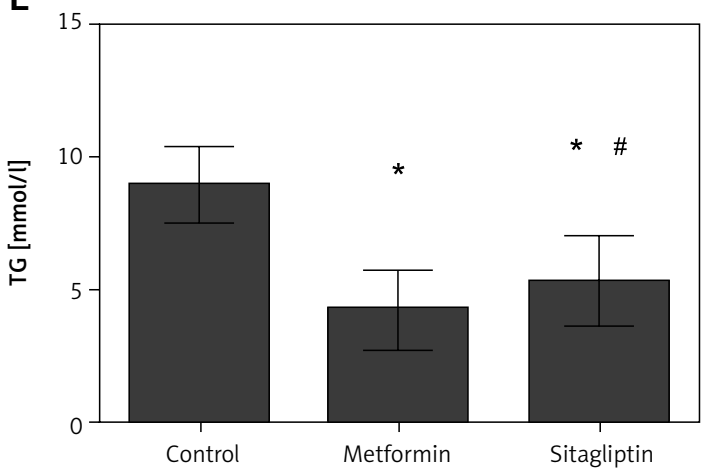

Our results agree with another study in healthy volunteers [24]. Therefore, for patients taking high carbohydrate food, sitagliptin may be superior to metformin.

In the high-lipid group, postprandial glucose and TG level were the highest in the four groups. The glucagon level in this group was lower but the insulin level was higher than any of the other groups, which suggests that a high fat diet induces significant insulin resistance. It was found that overnight infusion of $20 \%$ intralipid in healthy adolescents resulted in significantly increased fasting insulin and C-peptide levels and decreased hepatic and peripheral insulin sensitivity. A 40\% reduction in glucose uptake in the soleus muscle was noted after a 4-h intralipid infusion in another study in healthy adults. By contrast, acutely normalizing the chronically elevated plasma free fatty acid (FFA) levels with acipimox, a long-acting
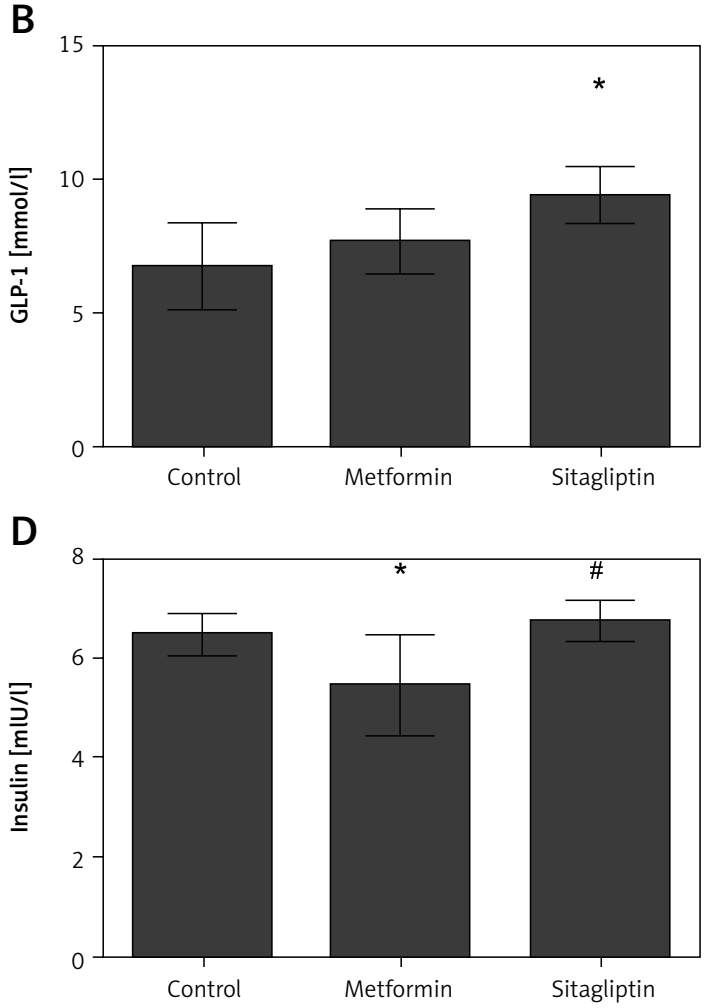

Figure 4. Effects of metformin and sitagliptin on glycolipid metabolism after high lipid diet. Rats in metformin groups were gavaged by $200 \mathrm{mg} / \mathrm{kg}$ metformin or $10 \mathrm{mg} / \mathrm{kg}$ sitagliptin before being gavaged by $45 \%$ glucose ( $4.5 \mathrm{ml} / \mathrm{kg}$, HCD group), $20 \%$ lipid emulsion ( $4.5 \mathrm{ml} / \mathrm{kg}$, HLD group), $20 \%$ lactalbumin (10 ml/kg, HPD group) or mixed diet (1: $1: 1$ of above diet ingredients, MD group). The energy was $33 \mathrm{~kJ}$ for all the diets

$H C D$ - high carbohydrate diet, HLD - high lipid diet, HPD - high protein diet, MD - mixed diet; * compared with control group $p<0.05$; ${ }^{*}$ compared with metformin group $p<0.05$.

antilipolytic drug, resulted in a two-fold increase in insulin sensitivity and a $30 \%$ decrease in glucose and insulin area under the curve during an oral glucose tolerance test in obese subjects with and without type 2 diabetes. Taken together, these observations provide evidence that acute increased plasma FFA level is an important contributor to the development of insulin resistance in healthy individuals and obese patients with and without T2DM [25-27]. Although the mechanism is not fully clarified, apparently any medicine targeting insulin resistance might be helpful in lowering postprandial glucose and TG level. In our study, we found although metformin and sitagliptin had a similar effect on P2BG, metformin had a profound role in lowering the TG level. In the metformin group, although the GLP-1 and glucagon did not change, the insulin level decreased significantly compared with the control group and sitagliptin group. Met- 
A

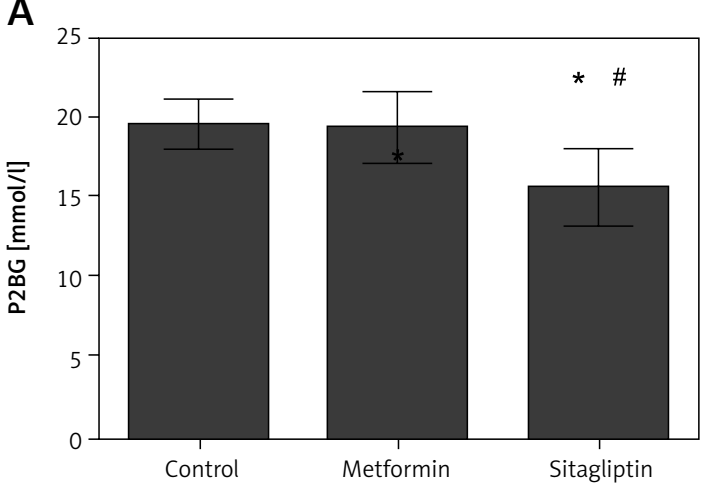

C

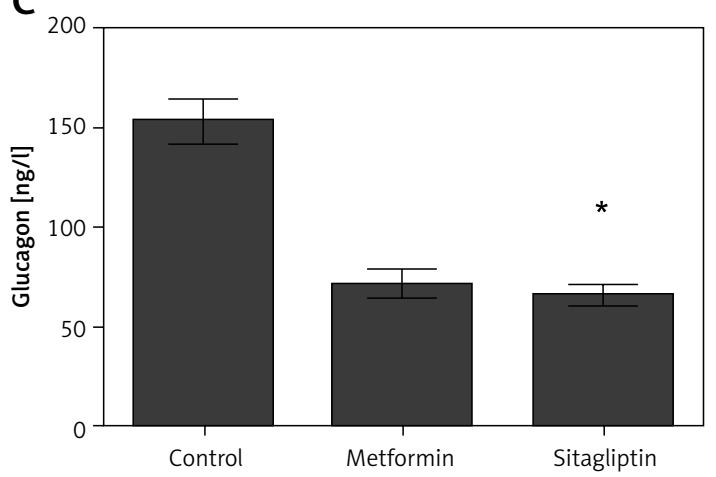

E

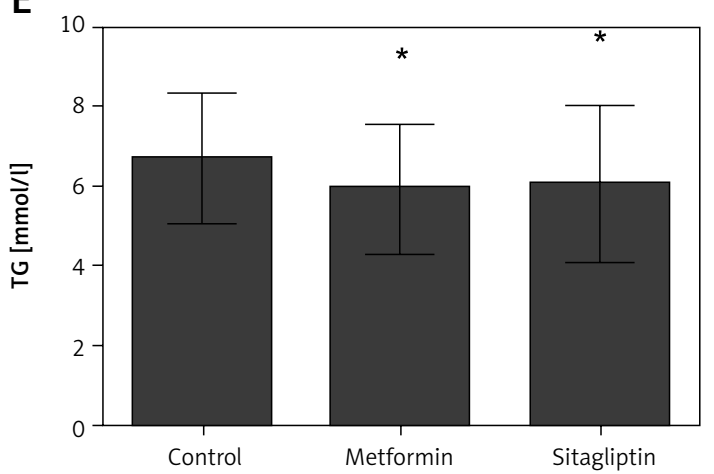

formin is generally recommended as first-line treatment in type 2 diabetes, especially in overweight patients. Metformin has been found to be safe and efficacious both as monotherapy and in combination with all oral antidiabetic agents and insulin. Moreover, metformin is increasingly used in non-alcoholic fatty liver disease, polycystic ovary syndrome and cancer [28]. Metformin reduces hyperglycemia primarily by inhibiting hepatic gluconeogenesis. It also increases insulin sensitivity, enhances peripheral glucose uptake (by inducing the phosphorylation of GLUT4 enhancer factor), and reduces insulin-induced suppression of fatty acid oxidation [29, 30]. Although sitagliptin also reduced P2BG and TG in the high lipid group, sitagliptin treatment lead to a further increase of insulin level compared with the control group, which might aggravate insulin resistance in the high-lipid group. Therefore, for a high fat diet, metformin
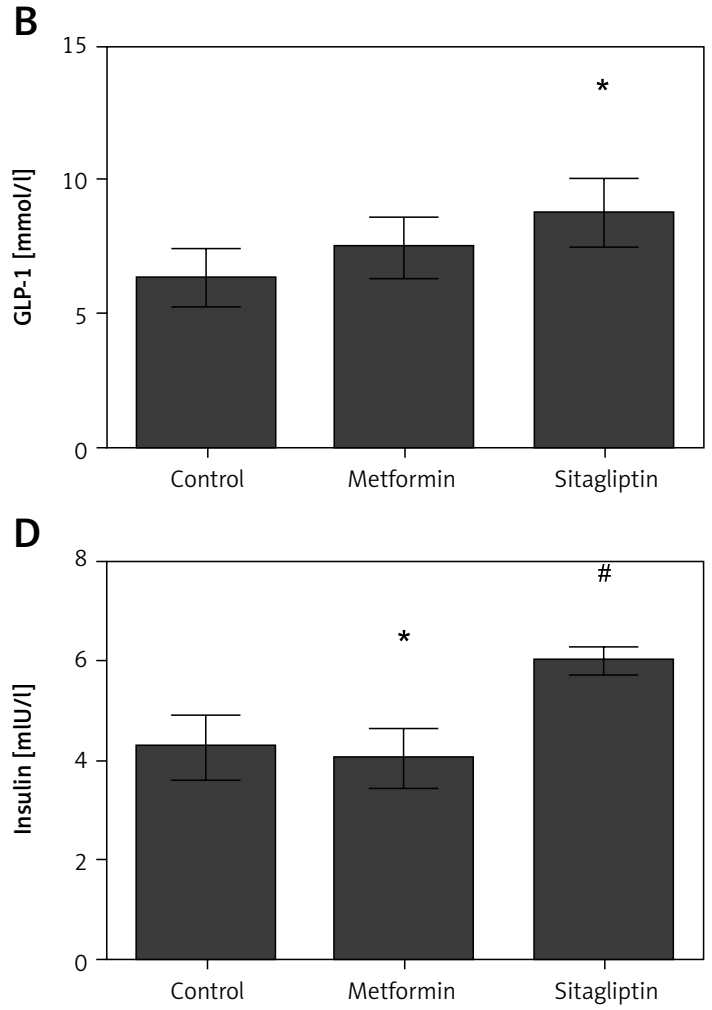

Figure 5. Effects of metformin and sitagliptin on glycolipid metabolism after high protein diet. Rats in metformin groups were gavaged by $200 \mathrm{mg} / \mathrm{kg}$ metformin or $10 \mathrm{mg} / \mathrm{kg}$ sitagliptin before gavaged by $45 \%$ glucose ( $4.5 \mathrm{ml} / \mathrm{kg}$, HCD group), $20 \%$ lipid emulsion ( $4.5 \mathrm{ml} / \mathrm{kg}$, HLD group), 20\% lactalbumin (10 ml/kg, HPD group) or mixed diet (1:1:1 of above diet ingredients, MD group). The energy was $33 \mathrm{~kJ}$ for all the diets

$H C D$ - high carbohydrate diet, HLD - high lipid diet, HPD - high protein diet, $M D$ - mixed diet; *compared with control group $p<0.05$; " compared with metformin group $p<0.05$.

is more beneficial in improving postprandial glycolipid metabolism and insulin resistance.

In the high protein group, the postprandial glucose and lipid levels were the lowest among the four groups. The glucagon level was significantly higher than that in other groups. This significant increase in glucagon would be expected to result in stimulation of gluconeogenesis and glycogenolysis and a subsequent increase in circulating plasma glucose concentrations. However, the glucose response was smaller with the high protein diet. The mechanism is not clear. The delayed gastric emptying, which will affect incretin secretion, may be a reason. Although in our study we did not find a significant difference of GLP-1 level between different diets, this cannot be applied to other incretin such as gastric inhibitory polypeptide (GIP) [31, 32]. In our study, we found that sitagliptin was effective in reducing both 
A

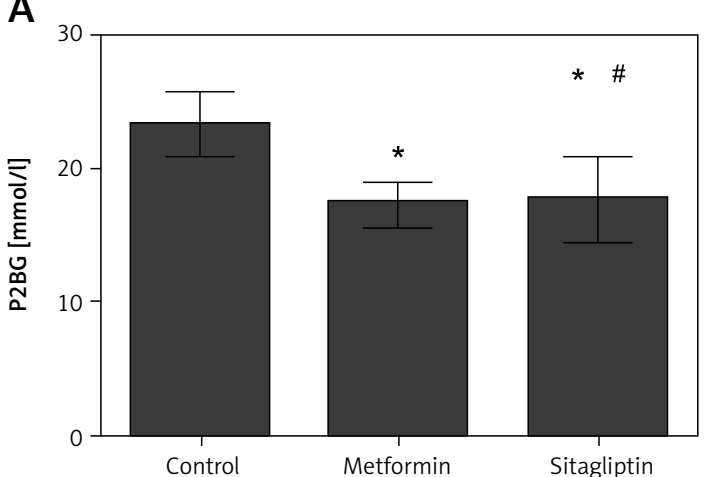

C

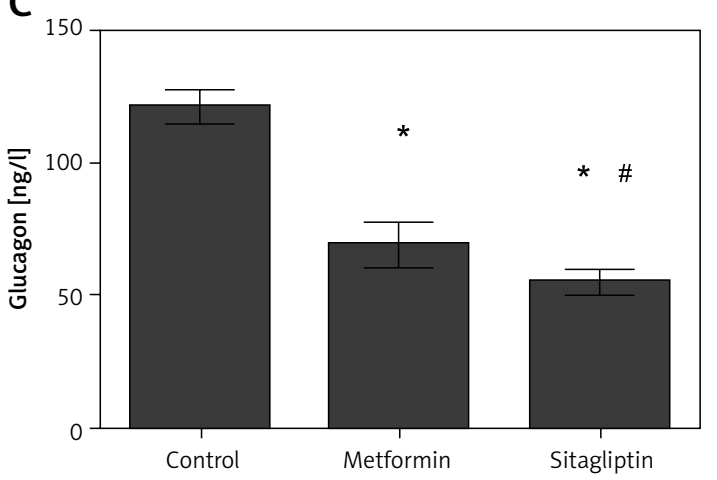

E

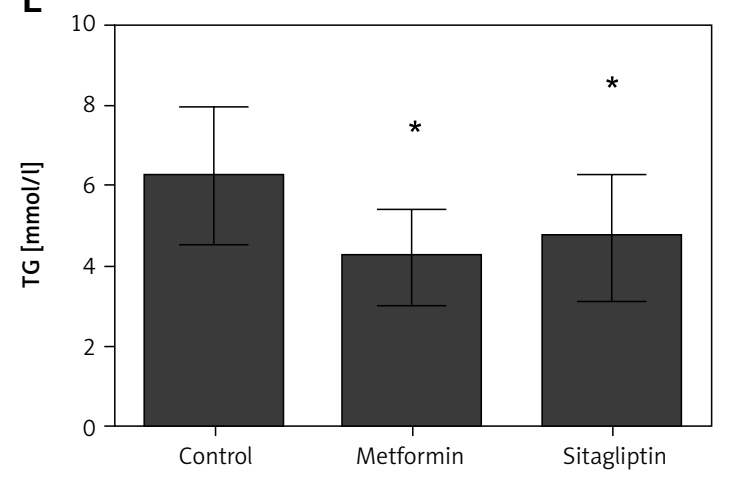

postprandial glucose and lipid levels by promoting insulin and the GLP-1 level and by inhibiting the glucagon level. However, it is interesting that metformin treatment did not reduce the P2BG level, although it did reduce the TG level after a high protein diet. The molecular mechanism of metformin is incompletely understood. AMPK is an enzyme that plays an important role in insulin signaling and in the regulation of whole body energy balance [33]. A study in 2001 suggested that activation of AMPK was required for metformin's inhibitory effect on the production of glucose by liver cells [34]. Research published in 2008 further showed that activation of AMPK was required for an increase in the expression of SHP, which in turn inhibited the expression of the hepatic gluconeogenic genes PEPCK and Glc-6-Pase [35]. However, researchers found that in rats fed a high protein diet for 14 days, the hepatic p-AMPKa level was

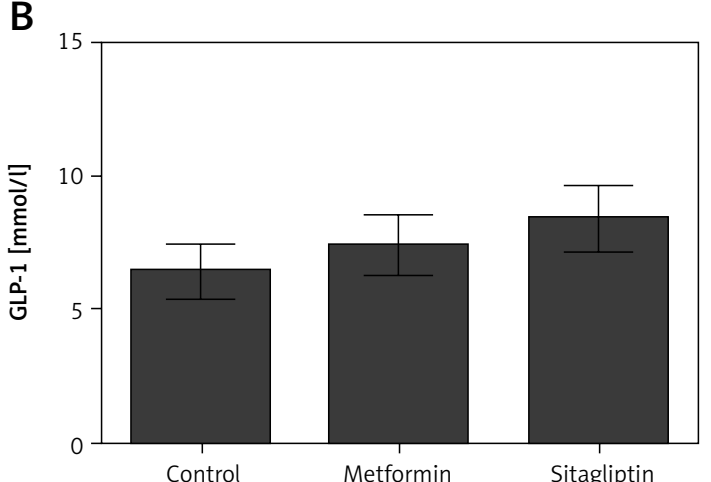

D

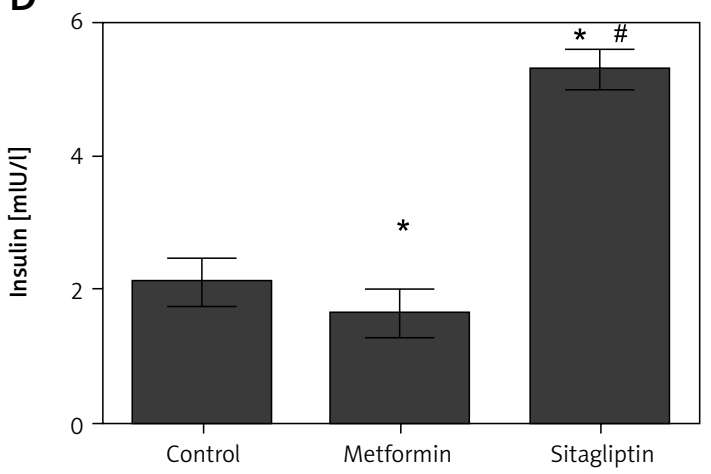

Figure 6. Effects of metformin and sitagliptin on glycolipid metabolism after mixed diet. Rats in metformin groups were gavaged by $200 \mathrm{mg} / \mathrm{kg}$ metformin or $10 \mathrm{mg} / \mathrm{kg}$ sitagliptin before gavaged by $45 \%$ glucose ( $4.5 \mathrm{ml} / \mathrm{kg}$, HCD group), $20 \%$ lipid emulsion (4.5 ml/kg, HLD group), 20\% lactalbu$\min (10 \mathrm{ml} / \mathrm{kg}, \mathrm{HPD}$ group) or mixed diet $(1: 1: 1$ of above diet ingredients, MD group). The energy was $33 \mathrm{~kJ}$ for all the diets

$H C D$ - high carbohydrate diet, HLD - high lipid diet, HPD - high protein diet, MD - mixed diet; *compared with control group $p<0.05$; ${ }^{*}$ compared with metformin group $p<0.05$.

lower than in rats receiving a normal protein diet. In primary cultured hepatocytes, a high concentration of amino acids reduced AMPK $\alpha$ phosphorylation [35]. In another study, it was found that a high protein diet reduced AMPK activity in the hypothalamus in normal rats and in ob/ob mice [36]. Therefore, a high protein diet may counteract the activation of AMPK by metformin. However, in our study, we used whey protein to mimic a high protein diet, and further studies are needed to determine whether these results can be applied to other proteins.

In a mixed diet (containing 33.3\% carbohydrate, $33.3 \%$ protein, $33.3 \%$ lipids), the P2BG was lower than that in the high carbohydrate group and the high lipid group, and just a little higher than the high protein group; the TG level was lower than other groups. The insulin level was lower than that in other groups. Therefore, a mixed diet 
is good for the control of glycolipid metabolism, and also causes fewer burdens on islet function. It is reasonable to recommend a balanced diet for type 2 diabetic patients. In this group, metformin was more efficient in controlling P2BG and TG levels compared with sitagliptin.

However, our study has a limitation in using single dietary ingredients to represent different diets, considering the large variability of different diet structures or different foods. Second, we used glucose to represent a high carbohydrate diet, used lipid emulsion to represent a high fat diet and used whey protein to represent a high protein diet; our results may not be applied to other ingredients. Therefore, more studies are needed in order to gain more precise results for the patients to choose the most suitable medicine according to different foods.

In conclusion, in the type 2 diabetic model, metformin and sitagliptin have different effects on glycolipid metabolism after different diets. If it is proved in type 2 diabetic patients, then different medicines may be recommended according to different diets in order to improve glycolipid metabolism.

\section{Acknowledgments}

Juhong Yang and Tu Ba contributed equally to this work.

This work was supported by the National Nature Science Foundation of China (no. 81273915, 81173428, 81200612, 81373864, 81473472).

\section{Conflict of interest}

The authors declare no conflict of interest.

\section{References}

1. Guariguata L. By the numbers: new estimates from the IDF Diabetes Atlas Update for 2012. Diabetes Res Clin Pract 2012; 98: 524-5.

2. Whiting DR, Guariguata L, Weil C, Shaw J. IDF diabetes atlas: global estimates of the prevalence of diabetes for 2011 and 2030. Diabetes Res Clin Pract 2011; 94: 311-21.

3. Guariguata L. Contribute data to the 6th edition of the IDF Diabetes Atlas. Diabetes Res Clin Pract 2013; 100: 280-1.

4. IDF Diabetes Atlas Group. Update of mortality attributable to diabetes for the IDF Diabetes Atlas: estimates for the year 2011. Diabetes Res Clin Pract 2013; 100: 277-9.

5. Lindi VI, Uusitupa MI, Lindström J, et al. Association of the Pro12Ala polymorphism in the PPAR-gamma2 gene with 3-year incidence of type 2 diabetes and body weight change in the Finnish Diabetes Prevention Study. Diabetes 2002; 51: 2581-6.

6. Papaetis GS, Papakyriakou P, Panagiotou TN. Central obesity, type 2 diabetes and insulin: exploring a pathway full of thorns. Arch Med Sci 2015; 11: 463-82.

7. Grant SF, Thorleifsson G, Reynisdottir I, et al. Variant of transcription factor 7-like 2 (TCF7L2) gene confers risk of type 2 diabetes. Nat Genet 2006; 38: 320-3.
8. Derosa G, Limas CP, Macías PC, et al. Dietary and nutraceutical approach to type 2 diabetes. Arch Med Sci 2014; 10: 336-44.

9. Franz MJ, Powers MA, Leontos C, et al. The evidence for medical nutrition therapy for type 1 and type 2 diabetes in adults. J Am Diet Assoc 2010; 110: 1852-89.

10. Nathan DM, Buse JB, Davidson MB, et al. Management of hyperglycemia in type 2 diabetes: a consensus algorithm for the initiation and adjustment of therapy: a consensus statement from the American Diabetes Association and the European Association for the Study of Diabetes. Diabetes Care 2006; 29: 1963-72.

11. American Diabetes Association, Bantle JP, Wylie-Rosett J, Albright AL, et al. American Diabetes Association. Nutrition recommendations and interventions for diabetes: a position statement of the American Diabetes Association. Diabetes Care 2008; 31 (Suppl. 1): S61-78.

12. Savory LA, Griffin SJ, Williams KM, et al. Changes in diet, cardiovascular risk factors and modelled cardiovascular risk following diagnosis of diabetes: 1-year results from the ADDITION-Cambridge trial cohort. Diabet Med 2014; 31: 148-55.

13. Krebs JD, Bell D, Hall R, et al. Improvements in glucose metabolism and insulin sensitivity with a low-carbohydrate diet in obese patients with type 2 diabetes. J Am Coll Nutr 2013; 32: 11-7.

14. Pagkalos EM. Combinations of insulin and oral hypoglycemic agents in diabetes mellitus type 2. Diabetes Res Clin Pract 2011; 93: S100-1.

15. Qaseem A, Humphrey LL, Sweet DE, Starkey M, Shekelle P; Clinical Guidelines Committee of the American College of Physicians. Oral pharmacologic treatment of type 2 diabetes mellitus: a clinical practice guideline from the American College of Physicians. Ann Intern Med 2012; 156: 218-31.

16. Freeman JS, Horton ES. What have we learned about the treatment of type 2 diabetes? The evolving paradigms. Am J Ther 2012; 19: 449-64.

17. Bi Y, Cai M, Liang H, et al. Increased carnitine palmitoyl transferase 1 expression and decreased sterol regulatory element-binding protein $1 \mathrm{c}$ expression are associated with reduced intramuscular triglyceride accumulation after insulin therapy in high-fat-diet and streptozotocin-induced diabetic rats. Metabolism 2009; 58: 779-86.

18. Raskin P. Sodium-glucose cotransporter inhibition: therapeutic potential for the treatment of type 2 diabetes mellitus. Diabetes Metab Res Rev 2013; 29: 347-56.

19. Maiese K, Chong ZZ, Shang YC, Wang S. Novel directions for diabetes mellitus drug discovery. Expert Opin Drug Discov 2013; 8: 35-48.

20. Hardman TC, Dubrey SW. New therapies in the management of type 2 diabetes mellitus. Br J Hosp Med (Lond) 2013; 74: 202-7.

21. Qaseem A, Humphrey LL, Sweet DE, Starkey M, Shekelle P; Clinical Guidelines Committee of the American College of Physicians. Oral pharmacologic treatment of type 2 diabetes mellitus: a clinical practice guideline from the American College of Physicians. Ann Intern Med 2012; 156: 218-31.

22. Zhu Q, Tong Y, Wu T, Li J, Tong N. Comparison of the hypoglycemic effect of acarbose monotherapy in patients with type 2 diabetes mellitus consuming an Eastern or Western diet: a systematic meta-analysis. Clin Ther 2013; 35: 880-99.

23. Ohlsson L, Alsalim W, Carr RD, et al. Glucose-lowering effect of the DPP-4 inhibitor sitagliptin after glucose 
and non-glucose macronutrient ingestion in non-diabetic subjects. Diabetes Obes Metab 2013; 15: 531-7.

24. Chia CW, Egan JM. Incretin-based therapies in type 2 diabetes mellitus. J Clin Endocrinol Metab 2008; 93: 3703-16.

25. Lee S, Boesch C, Kuk JL, Arslanian S. Effects of an overnight intravenous lipid infusion on intramyocellular lipid content and insulin sensitivity in African-American versus Caucasian adolescents. Metabolism 2013; 62: 417-23.

26. Burns SF, Kelsey SF, Arslanian SA. Effects of an intravenous lipid challenge and free fatty acid elevation on in vivo insulin sensitivity in African American versus Caucasian adolescents. Diabetes Care 2009; 32: 355-60.

27. Black MH, Watanabe RM, Trigo E, et al. High-fat diet is associated with obesity-mediated insulin resistance and beta-cell dysfunction in Mexican Americans. J Nutr 2013; 143: 479-85.

28. Cicero AF, Tartagni E, Ertek S. Metformin and its clinical use: new insights for an old drug in clinical practice. Arch Med Sci 2012; 8: 907-17.

29. Collier CA, Bruce CR, Smith AC, Lopaschuk G, Dyck DJ. Metformin counters the insulin-induced suppression of fatty acid oxidation and stimulation of triacylglycerol storage in rodent skeletal muscle. Am J Physiol Endocrinol Metab 2006; 291: E182-9.

30. Chotechuang N, Azzout-Marniche D, Bos C, et al. mTOR, AMPK, and GCN2 coordinate the adaptation of hepatic energy metabolic pathways in response to protein intake in the rat. Am J Physiol Endocrinol Metab 2009; 297: E1313-23.

31. Veldhorst M, Smeets A, Soenen S, et al. Protein-induced satiety: effects and mechanisms of different proteins. Physiol Behav 2008; 94: 300-7.

32. Moran TH, Dailey MJ. Intestinal feedback signaling and satiety. Physiol Behav 2011; 105: 77-81.

33. Towler MC, Hardie DG. AMP-activated protein kinase in metabolic control and insulin signaling. Circ Res 2007; 100: 328-41.

34. Zhou G, Myers R, Li Y, et al. Role of AMP-activated protein kinase in mechanism of metformin action. J Clin Invest 2001; 108: 1167-74.

35. Kim YD, Park KG, Lee YS, et al. Metformin inhibits hepatic gluconeogenesis through AMP-activated protein kinase-dependent regulation of the orphan nuclear receptor SHP. Diabetes 2008; 57: 306-14.

36. Ropelle ER, Pauli JR, Fernandes MF, et al. A central role for neuronal AMP-activated protein kinase (AMPK) and mammalian target of rapamycin (mTOR) in high-protein diet-induced weight loss. Diabetes 2008; 57: 594-605. 